\title{
Research on Thermal Conductivity of Nanopaper Enabled Composite Materials
}

\author{
Aying Zhang ${ }^{1, a,{ }^{*}}$, Zhenghong $\mathrm{Li}^{2, \mathrm{~b}}$ \\ ${ }^{1}$ Harbin University, 150086 Harbin, China \\ ${ }^{2}$ Harbin Institute of Technology, 150001 Harbin, China \\ a,*zaying@sina.com, b273662999@qq.com
}

Keywords: FLUENT, Simulation, Nanopaper, Composites.

Abstract. FLUENT was used to analyze how the shapes of nanopaper affect the temperature distribution, the changes of the temperature with time and the heating rate during heating. The obtained temperature distribution maps shows that high temperature region appear in the vicinity of the nanopaper. The curve of the average temperature exhibit similar trend and there are two distinct stages observed. The first stage shows that the slopes of the curves increase significantly with increasing the heating time. A progressive but slow increase of the average temperature is observed in the second stage.

\section{Introduction}

Carbon nanotubes (CNTs) have been comprehensively studied for their excellent electrical, chemical, thermal and mechanical properties [1-4]. Compared with graphite films, due to the excellent thermal conductivity, the polymer composites reinforced by the nanopaper can be used to solve the heat problems associated with advanced electronic equipment [5-10]. Studies on their thermal properties are less frequently reported, particularly for the thermal properties of nanopaper reinforced polymer composites analyzed by using the finite element analysis. In this study, heating model of the polymer composite reinforced by different shape nanopaper is established to predict the thermal property of nanopaper reinforced composite.

\section{Numerical model}

The finite element software FLUENT was used to simulate the thermal property of the polymer composites reinforced by sinusoidal and pulse bending nanopaper. The heating model of the polymer composite reinforced by the nanopaper is shown in Figure 1.

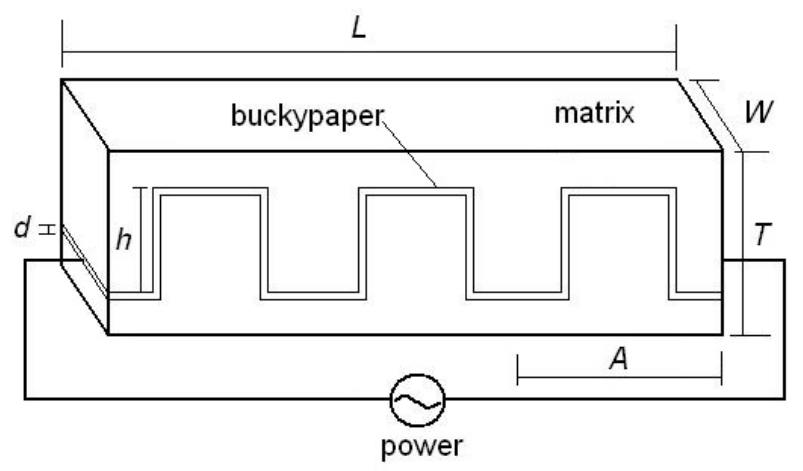

Fig. 1. Sketch diagram of heating experimental device

The length $(L)$, width $(w)$, and the thickness $(T)$ of the heating model of the polymer composite reinforced by both sinusoidal and pulse bending nanopaper are $600 \mathrm{~mm}, 50 \mathrm{~mm}$, and $100 \mathrm{~mm}$ 
respectively. The thicknesses $(h)$ of sinusoidal and pulse bending nanopaper are $10 \mathrm{~mm}$. The bending height $(h)$ and bending period $(A)$ of pulse bending nanopaper are $60 \mathrm{~mm}$ and $120 \mathrm{~mm}$. In Figure 1 , heating the nanopaper is driven by a power source, and the cube region is the polymer matrix which is heated by the nanopaper. The temperature of the polymer matrix is increased due to the electro-heating of the nanopaper.

The objective of this research is to analyze the effect of the shapes of nanopaper on the changes of the average temperature with time and the heating rate when the composite materials is heated using a finite element software FLUENT. The changes of the average temperature with time and the heating rate were analyzed when the composite materials in the heating process until reaching the steady state. During the analysis, two different shapes of nanopaper were considered: sinusoidal and pulse bending; the heating powers were varied from 5,25 , to $50 \mathrm{~W}$; the nanopaper were $1 \mathrm{~W} /(\mathrm{m} \bullet \mathrm{K})$; the thermal conductivity of the polymer matrix were varied from $0.02,0.05,0.10$, and $0.20 \mathrm{~W} /(\mathrm{m} \bullet \mathrm{K})$.

Boundary conditions were pre-seted as follow. The natural convection heat transfer coefficient was set to be $10 \mathrm{~W} /\left(\mathrm{m}^{2} \bullet \mathrm{K}\right)$. The ambient temperature was set to be $300 \mathrm{~K}$. The thermal conductivity of nanopaper was set to be $1.0 \mathrm{~W} /(\mathrm{m} \cdot \mathrm{K})$. The specific heat capacity of nanopaper was set to be 1000 $\mathrm{J} /(\mathrm{kg} \cdot \mathrm{K})$. The density of nanopaper was set to be $500 \mathrm{~kg} / \mathrm{m}^{3}$. The thermal conductivity of the polymer matrix was set to be $0.1 \mathrm{~W} /(\mathrm{m} \bullet \mathrm{K})$. The specific heat capacity of the polymer matrix was set to be 1300 $\mathrm{J} /(\mathrm{kg} \cdot \mathrm{K})$. The density of the polymer matrix was set to be $1000 \mathrm{~kg} / \mathrm{m}^{3}$.

Figure 2 and Figure 3 shows the geometry model of the polymer composites reinforced by sinusoidal and pulse bending nanopaper.

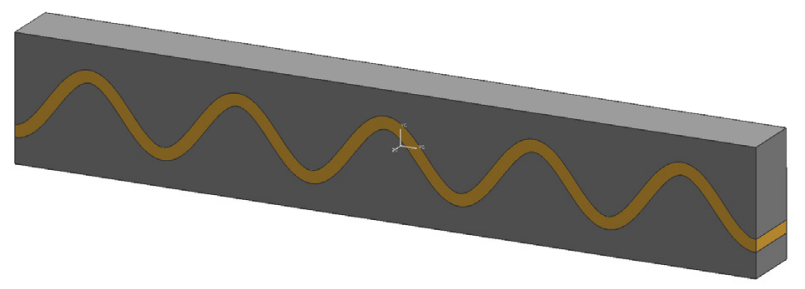

Fig. 2. Model of the polymer composites reinforced by sinusoidal nanopaper

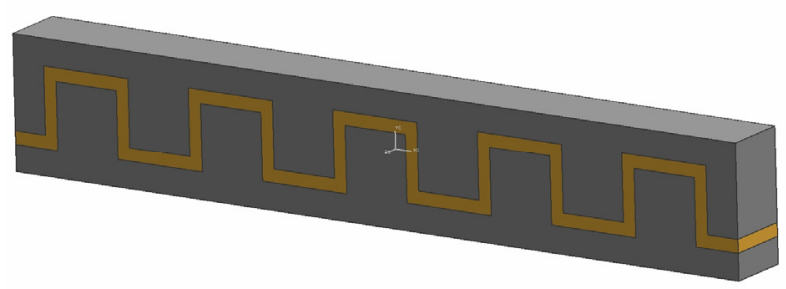

Fig. 3. Model of the polymer composites reinforced by pulse bending nanopaper

The inner heat source of the nanopaper with sinusoidal heating sheets can be obtained by formula (1).

$$
\Phi_{\text {sin }}=\frac{25 \mathrm{~W}}{(0.01 \times 0.05 \times 0.161226) \mathrm{m}^{3} \times 5}=62024.7 \mathrm{~W} / \mathrm{m}^{3}
$$

The inner heat source of the nanopaper with pulse bending heating sheets can be obtained by formula (2).

$$
\Phi_{\text {pulse }}=\frac{25 \mathrm{~W}}{(0.01 \times 0.05 \times 0.22) \mathrm{m}^{3} \times 5}=45454.5 \mathrm{~W} / \mathrm{m}^{3}
$$




\section{Results and discussion}

The temperature of the polymer composites reinforced by sinusoidal nanopaper at stable states along the section $\mathrm{x}=0$ are listed in Table 1 .

Table 1 Temperature of composites reinforced by sinusoidal nanopaper along the sections $\mathrm{x}=0$

\begin{tabular}{ccc}
\hline $\mathrm{T}_{\max } / \mathrm{K}$ & $\mathrm{T}_{\min } / \mathrm{K}$ & $\mathrm{T}_{\mathrm{ave}} / \mathrm{K}$ \\
\hline 349.586 & 302.350 & 325.822 \\
\hline
\end{tabular}

The temperature distribution of the polymer composites reinforced by sinusoidal nanopaper at the stable states along the section $\mathrm{x}=0$ were calculated. The obtained temperature distribution maps are shown in Figures 4. Clearly, high temperature region appear in the vicinity of the nanopaper.

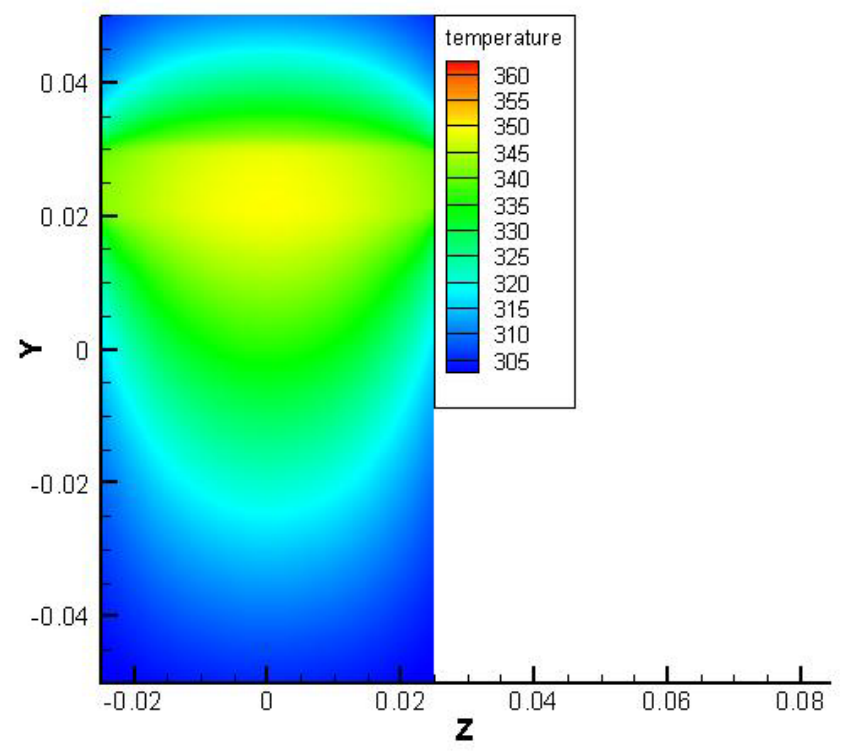

Fig. 4. Temperature distribution maps of composites reinforced by sinusoidal nanopaper along the section $\mathrm{x}=0$

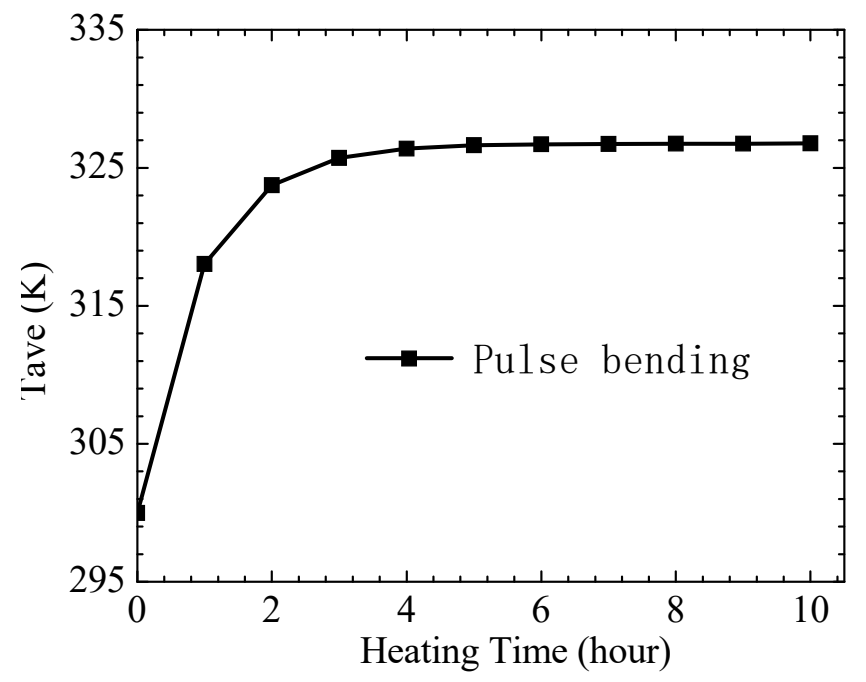

Fig. 5. Temperature distribution maps of composites reinforced by pulse bending nanopaper along the section $\mathrm{z}=0$

The heating rate and the time for the nanopaper/polymer composites reaching a steady state were calculated by the finite element software FLUENT. The relationship of the average temperature as a function of time in a transient state along the section $\mathrm{z}=0$ was also analyzed and the results are shown in Figure 5. Figure 5 shows that the curve of the average temperature exhibit similar trend and there are two distinct stages observed. The first stage shows that the slopes of the curves increase 
significantly with increasing the heating time. A fast increase of the average temperature occurs during stage I after which the slope is changed, indicating the beginning of the second stage. A progressive but slow increase of the average temperature is observed in the second stage.

\section{Summary}

FLUENT was used to analyze how the shapes of nanopaper affect the temperature distribution, the changes of the temperature with time and the heating rate during heating. The obtained temperature distribution maps shows that high temperature region appear in the vicinity of the nanopaper. The curve of the average temperature exhibit similar trend and there are two distinct stages observed. The first stage shows that the slopes of the curves increase significantly with increasing the heating time. A progressive but slow increase of the average temperature is observed in the second stage.

\section{Acknowledgement}

This research were financially supported by Heilongjiang Natural Science Foundation (Grant No. E201454) and Heilongjiang Postdoctoral Scientific Research Developmental Fund (Grant No. LBH-Q16141).

\section{References}

[1] R. S. Ruoff and D. C. Lorents, Mechanical and thermal properties of carbon nanotubes, Carbon, vol. 33, pp. 925-930, 1995.

[2] J. Hone and J. E. Fischer, Quantized phonon spectrum of single-wall carbon nanotubes, Science, vol. 289, pp. 1730-1733, 2000.

[3] S. Berber, Y. K. Kwon and D. Tomanek, Unusually high thermal conductivity of carbon nanotubes, Physical Review Letters, vol. 84, pp. 4613-4616, 2000.

[4] M. A. Osman and D. Srivastava, Temperature dependence of the thermal conductivity of single-wall carbon nanotubes, Nanotechnolgy, vol. 12, pp. 21-24, 2001.

[5] P. Kim, L. Shi, A. Majumdar and P. L. McEuen, Thermal transport measurements of individual multiwalled nanotubes, Physical Review Letters, vol. 87, pp. 265-266, 2001.

[6] M. Fujii, X. Zhang, H. Xie and et al, Measuring the thermal conductivity of a single carbon nanotube, Physical Review Letters, vol. 95, pp. 065502, 2005.

[7] Q. Li, C. Liu, X. Wang and et al, Measuring the thermal conductivity of individual carbon nanotubes by the Raman shift method, Nanotechnology, vol. 20, pp. 5886-5890, 2009.

[8] A. A. Balandin, Thermal properties of graphene and nanostructured carbon materials, Nature Materials, vol. 10, pp. 569-581, 2011.

[9] E. Pop, D. Mann, Q. Wang and et al, Thermal conductance of an individual single-wall carbon nanotube above room temperature, Nano Letter, vol. 6, pp. 96-100, 2005.

[10] C. Yu, L. Shi and et al, Thermal conductance and thermopower of an Individual Single-Wall Carbon Nanotube, Nano Letter, vol. 5, pp.1842-1846, 2005. 